\title{
Atomistically Informed Stochastic Multiscale Model to Predict the Behavior of Carbon Nanotube - Enhanced Nanocomposites
}

\author{
Nithya Subramanian ${ }^{1}$, Ashwin Rai ${ }^{1}$, and Aditi Chattopadhyay ${ }^{1} *$
} Arizona State University, 551 E. Tyler Mall, Tempe, AZ, 85287

\begin{abstract}
A comprehensive, point-information-to-continuum-level analysis framework is presented in this paper to accurately characterize the behavior of carbon nanotube (CNT)-enhanced composite materials. Molecular dynamics (MD) simulations are performed to study subnanoscale interactions of the CNT with the polymeric phase of the nanocomposite. The effect of cross-linking between the epoxy resin and the hardener on the mechanical properties of the polymer is investigated; furthermore, the effect of CNT weight fraction on the probability distribution of polymer cross-linking degree is also studied through stochastic models. The stochastic distributions obtained from MD simulations provide a basis to simulate local variations in the matrix properties in the continuum model at the microscale. The inclusion of an atomistically informed elastic-plastic model at the microscale reveals a significant deviation of the mechanical properties from those obtained based on classical homogenization approaches. Microstructural variability arising from heterogeneous cross-linking degree in the polymer phase and variations in fiber geometry and spacing is also found to cause deviations in the mechanical response when compared to the assumption of a perfectly ordered fiber-matrix microstructure.
\end{abstract}

\section{Introduction}

Carbon nanotubes (CNTs), due to their unique mechanical properties, have generated tremendous scientific and technical interest in the past decade [1, 2]. Nanocomposites comprising inorganic/organic nanoparticles dispersed in a polymer medium offer improved performance sought after in many engineering applications. CNT-enhanced composites have the potential to make significant improvements in strength, stiffness [3], corrosion resistance [4], and damage sensing [5], which are desirable characteristics to

* Corresponding Author: Tel: (480) 965 9342, Email: aditi@asu.edu (Aditi Chattopadhyay) 
aerospace applications. Although nanocomposites have been under development for several years, their implementation remains limited due to a lack of understanding of the basic physical relationships between nanoscale structural parameters and macroscale properties. A major challenge has been how to scale material behavior from the nanoand microscale to the macroscale to investigate the effect of the nanoparticle at the structural scale. Inherent to each length scale is variation and uncertainty in the constituent material properties and architecture. These variations at the constituent level may be significant enough to result in a stochastic response at the macroscale. Therefore, physics-based computational models offer key capabilities in predicting macroscale behavior as a function of stochastic nano- and microscale variability and properties.

To model polymeric systems integrated with nanoparticles, several computational approaches have been developed [6,7]. These include homogenization techniques, molecular dynamics (MD) simulation, Monte Carlo (MC) simulation and the MoriTanaka approach. Homogenization techniques [8-10] are limited to the microscale and are not suitable for describing the interface effect between nanoparticle and the matrix material. MD simulations study the interactions among a group of atoms or molecules and have been widely employed to understand the formation, breaking, and properties of cross-link bonds in polymers with appropriate force-field potentials [11]. Subramanian et al. [12] and Koo et al. [13] recently introduced an approach to stochastically simulate the chemical curing process in epoxy polymers through MD simulations. The authors studied the effect of the polymer cross-linking degree on the mechanical properties of a smart polymer. A review of modeling techniques to determine the mechanical properties of polymeric nanocomposites using MD and MC methods has been presented by Valavala and Odegard [14]. Li and Strachan [15] performed MD simulations to predict the thermomechanical response of an epoxy thermoset polymer by studying the role of various factors such as the degree of polymerization, strain rate, temperature, etc. An extensive review was presented by Meysam and Hubert [16] on the nanoscale interactions between CNTs and the polymer in nanocomposites by exploring both experimental and modeling techniques. The authors investigated experimental methods such as spectroscopy and probe microscopy to measure interaction forces and interfacial stresses between the constituent materials. They also examined modeling techniques ranging from quantum 
mechanics based approaches to molecular and coarse-grained models and concluded that MD model predictions for thermo-mechanical properties compared remarkably well with experimental measurements. Thus, the MD method has tremendously contributed to the study of interactions in multiphase materials. However, the high computational cost incurred by large-scale MD simulation restricts its use beyond the nanoscale. Micromechanical homogenization techniques have been widely implemented to compute the effective properties of polymer matrix composites at the microscale; however, such homogenization techniques in a multiscale framework can propagate large errors across length scales. Another critical aspect of multiscale approaches that has been investigated is the bridging of information across length scales, especially from the discrete atomistic level to continuum models. Subramaniyan and Sun [17] showed that sufficient spatial and temporal averaging of the atomistic virial stresses provided a good estimate of continuum level Cauchy stresses. The authors used multiple numerical examples with systems of about 3000 atoms and performed MD simulations with thermal loading under different ensembles to show that the averaged virial stress values converged to the continuum stress values. Hence, in this study, multiple equilibration stages are introduced in MD simulations on large atomic systems to obtain well-averaged values of virial stresses. This approach serves as a reliable bridging mechanism from the discrete nanoscale to the continuum microscale.

Most micromechanical modeling frameworks for polymer matrix composites assume a periodic and uniform arrangement of fibers. The effect of microstructural stochasticity arising from fiber packing has been investigated by a few researchers. Borkowski and coworkers [18] used a fiber-centered continuum model array and simulated the effects of random variations in fiber spacing and fiber diameter. Similar studies were conducted by Huang et al. [19] and Buryachenko et al. [20] on random fiber arrangements in unidirectional composites to simulate the effects of different loading angles and packing structure, respectively, on the overall mechanical properties. However, the properties of the polymer matrix were assumed to be uniform in the aforementioned studies. The conclusions from these studies suggest that the quantification of microstructure variability is critical when small length scale phenomena drive global response. Therefore, a holistic microstructural model needs to also quantify the influence of microstructural 
randomness arising from local variations in matrix properties on the effective homogenous response of the fiber-matrix system.

In this work, MD simulations simulate chemical cross-link formation between the resin and the hardener molecules resulting in a cured epoxy system. The cured epoxy polymer is further subjected to uniaxial deformation, triaxial compression, and plastic deformation to obtain relevant mechanical properties. The effect of the inclusion of CNTs in the matrix on the cross-linking of the polymer is studied and the corresponding probability distribution of cross-linking degrees is generated using statistical data from MD simulations. At the microscale, a fiber-centered continuum model simulates the variations in fiber diameter, fiber spacing, and weight fraction of CNT inclusions in the surrounding matrix. Thus, in this study, the results obtained from MD simulations are efficiently transferred to simulate local variations in matrix properties at the microscale. The elastic-plastic response of the CNT-dispersed matrix phase, determined from MD simulations, is used to define the post-yield hardening response at the microscale. Figure 1 illustrates the flow of information between the various models in the multiscale framework.

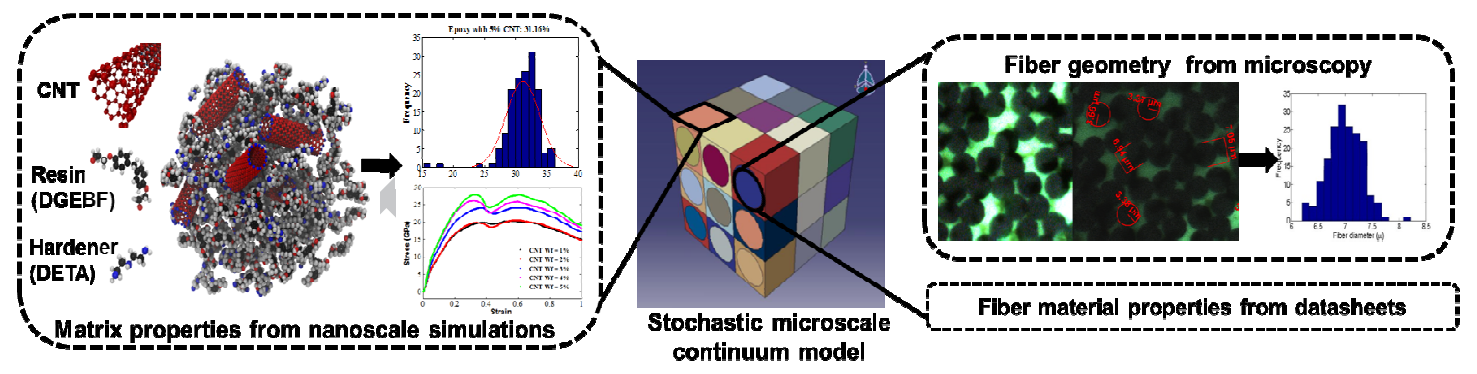

Figure 1. Schematic of multiscale modeling framework

\section{Nanoscale Modeling}

MD simulations are performed to characterize the linear and nonlinear material response of the nanopolymer at the molecular level. The simulation results account for pair-, bond-, charge-, and geometric distortion-related interactions described by a forcefield. These results are used as inputs to a continuum-based representative unit cell (RUC) at the microscale. The MD simulation package, Large-scale Atomic Molecular Massively Parallel Simulator (LAMMPS) [21], is used to perform the simulations. An all-atom 
force-field - OPLS (Optimized Potentials for Liquid Simulations) [22], is implemented to obtain the atomic and molecular interactions in the elastic region.

The molecular structures of the epoxy resin and the hardener that constitute the polymer used in this study are DGEBF (Di-Glycidyl Ether of Bisphenol F) and DETA (DiEthylene Tri-Amine), respectively. The ratio of constituents by weight in the nanoscale RUC containing neat epoxy is shown in Table 1. The term 'cross-linking degree' is equivalent to the degree of cure and it is calculated as the percentage of actual crosslinked bonds (between the resin and the hardener molecules) over all possible cross-link bonds to saturate the resin active sites. These cross-link bonds are an effective means of load transfer as they enable the polymer chains to entangle and form a polymer network; hence, it is critical to investigate the relationship between polymer cross-linking degree and the mechanical properties of interest.

Table 1. Ratio of nanoscale RUC constituents by weight

\begin{tabular}{|c|c|c|}
\hline Constituent & Weight $(\mathrm{g} / \mathrm{mol})$ & Chemical Formula \\
\hline DGEBF (epoxy resin) & 313 & $\mathrm{C}_{19} \mathrm{H}_{20} \mathrm{O}_{4}$ \\
\hline DETA (hardener) & 103 & $\mathrm{C}_{4} \mathrm{H}_{13} \mathrm{O}_{6}$ \\
\hline
\end{tabular}

A stochastic approach based on a cutoff distance between active sites in the resin and the hardener molecules is used to simulate the variability in cross-linking degree and mechanical properties of the polymer system. The epoxy resin molecule possesses two active sites for bond formation whereas the hardener molecule contains five active sites. A covalent bond is generated when the distance between the active sites of the epoxy resin and the hardener is shorter than a critical cutoff value. The probability distribution of cross-linking degree for the neat polymer is determined from $500 \mathrm{MD}$ simulations with random initial positions of molecules. The simulation implements periodic boundary conditions and an isobaric-isothermal (NPT) ensemble (300 K and $1 \mathrm{~atm})$ to equilibrate the system for $100 \mathrm{ps}$. Each RUC is cured using a bond formation command in LAMMPS and a cross-linking cutoff distance of 4.5 Angstroms; this cutoff value is selected because it is more than twice the length of a carbon-nitrogen covalent bond. The choice of this cutoff distance is also driven by the validation of resulting cross-linking degree from simulations with the experimentally measured degree of cure in the epoxy samples using digital scanning calorimetry (DSC). Figure 2 shows the resulting statistical distribution of 
cross-linking degree. Using the Kolmogorov-Smirnov (KS) test on the crosslinking degree, the maximum difference of the means is found to be 0.047 ; this value is much below the $99 \%$ confidence level KS parameter of 0.073 . Hence, the crosslinking degree is assumed to be distributed normally.

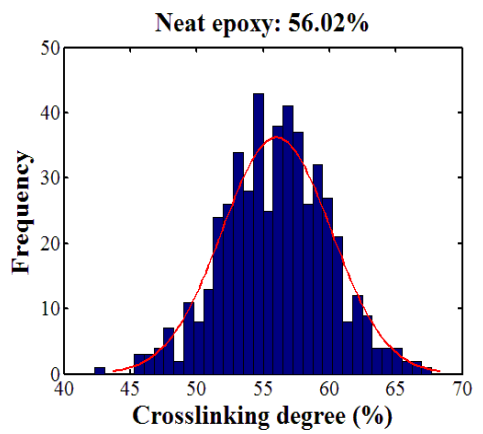

Figure 2. Distribution of most likely cross-linking degree in neat epoxy

The inclusion of CNT molecules in the polymer system is expected to interfere with the polymer curing process. This effect of CNT inclusion needs to be investigated because a polymer matrix with low cross-linking degree may not transfer loads effectively and therefore, could serve as a damage initiation site. Hence, a total of 150 MD simulations are performed to determine the most likely cross-linking degree of the polymer in the presence of CNT molecules in the system. Figure 3 illustrates the resulting probability distribution with the mean cross-linking degree value decreasing as the inclusion of CNTs by weight increases. For example, the most likely cross-linking degree is $31.16 \%$ at $5 \%$ CNT weight fraction and $17.35 \%$ at $10 \%$ CNT weight fraction.

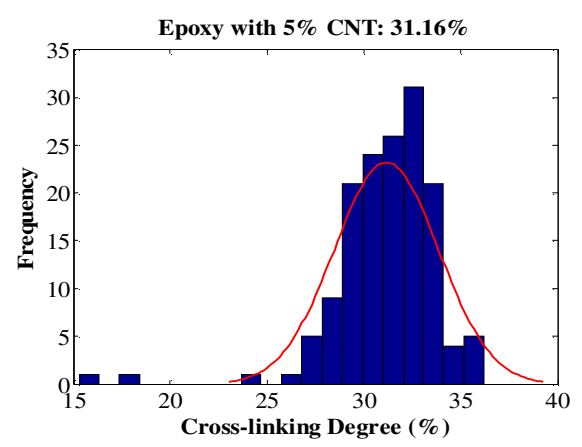

(a)

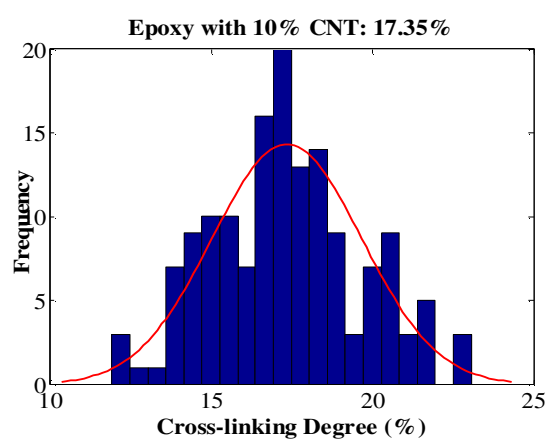

(b)

Figure 3. Distribution of most likely cross-linking degree in CNT-epoxy system (a) 5\% CNT weight fraction (b) $10 \%$ CNT weight fraction.

MD simulations are also performed on the cross-linked polymer systems to obtain the tensile and shear moduli. The equilibrated RUCs are deformed along the $\mathrm{x}, \mathrm{y}$, and $\mathrm{z}$ axes 
independently under the NPT ensemble ( $300 \mathrm{~K}$ and 0 atm). Based on the MD simulation results, the Young's modulus of the polymer system is calculated from the slope of the polymer stress-strain response in the linear elastic region. Triaxial deformation test simulations are conducted to determine the bulk modulus, $K$, based on Equation (1) by varying the hydrostatic pressure from 0 atm to 5000 atm $(\Delta p)$ at room temperature. The shear modulus, $G$, of the polymer system is calculated using Equation (2) under the assumption of isotropy, which is valid because the mechanical response of the polymer is found to be independent of the axis of deformation in a system with randomly oriented and well-dispersed CNTs. The variation of mechanical properties with change in crosslinking degree, as shown in Figure 4, appears to be linear. As the cross-linking degree increases, the matrix phase has higher stiffness and load carrying ability due to higher density of chemical bonds.

$$
\begin{aligned}
& K=\frac{\Delta p}{\Delta V / V_{0}} \\
& G=\frac{3 K E}{9 K-E}
\end{aligned}
$$

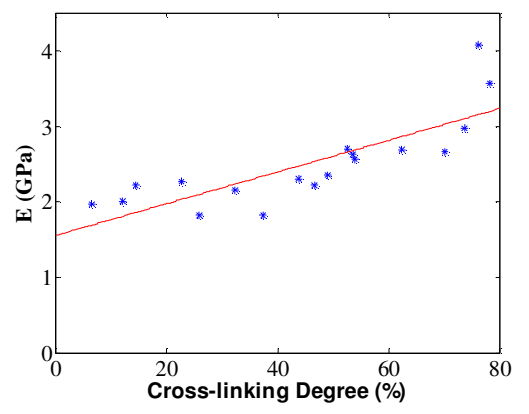

(a)

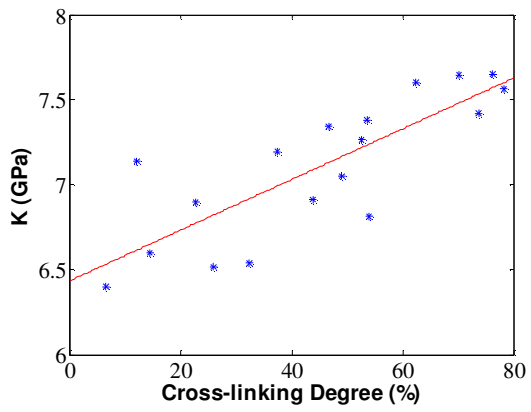

(b)

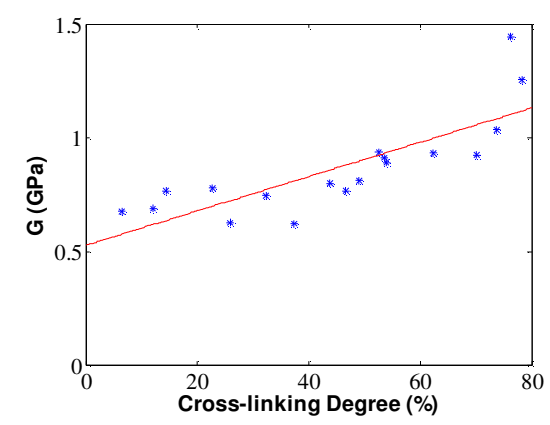

(c)

Figure 4. Variation of mechanical properties with cross-linking degree in neat polymer (a) Tensile modulus (b) Bulk modulus (c) Shear modulus 
MD simulations employed with the all-atom force field - OPLS are not capable of capturing bond dissociation between atoms leading to plasticity. Although MD simulations using OPLS are useful to study processes in a system around its equilibrium state (e.g. curing), they are not appropriate for investigating covalent bond dissociation occurring away from the equilibrium state (e.g. during deformation tests). However, the curing simulations represent near-equilibrium processes that can be accurately captured using the classical all-atom force field, which are much more computationally efficient than bond order based force fields. The external forces propagate through bonds in the matrix and in turn, the deformation induced by mechanical loading elongates covalent bonds. It is therefore of interest to capture the effective local force in the deformed thermoset matrix to identify covalent bond dissociation, which characterizes the plasticity in the system at higher length scales. Here, a reactive force field (ReaxFF), which is a bond order based potential, is employed to perform deformation tests on a nanoscale RUC and to observe the nonlinear response of the CNT epoxy system. The identification of optimal parameters for the bond order based potential that can accurately characterize the nonlinear response of this novel nanopolymer requires rigorous calculations involving density functional theory (DFT) arising from quantum mechanics. However, several bond order based potentials exist for organic systems and hydrocarbons [23-27]. The most appropriate bond order based potential for the CNT-enhanced polymer system under consideration is determined by a bond elongation test of a specific $\mathrm{C}-\mathrm{H}$ bond in a CNT with end-Hydrogen atoms followed by the estimation of the corresponding bond dissociation energy from MD simulations. The bond dissociation energy from different potentials is compared to the bond strength of the $\mathrm{C}-\mathrm{H}$ bond in the CNT calculated using DFT as reported by Wei et al. to be $-2.39 \mathrm{eV}$ (equivalent to $-55.11 \mathrm{kCal} / \mathrm{mol}$ ) [28]. Table 2 lists the bond dissociation energy calculated from MD simulations using different bond order based potential parameters.

Table 2. Comparison of bond dissociation energy

\begin{tabular}{|c|c|}
\hline $\begin{array}{c}\text { Bond order based } \\
\text { potential }\end{array}$ & $\begin{array}{c}\text { Bond dissociation } \\
\text { energy }(\mathrm{kCal} / \mathrm{mol})\end{array}$ \\
\hline Chenoweth et al. [24] & -86.45 \\
\hline Weismiller et al. [25] & -89.66 \\
\hline Singh et al. [26] & -57.04 \\
\hline Mattsson et al. [27] & -145.22 \\
\hline
\end{tabular}


The RUC shown in Figure 5 contains a single CNT oriented along the loading direction (i.e., x-axis). Therefore, the simulation volume represents the best possible load transfer mechanism as CNTs can carry maximum load along their longitudinal axis. Figure 6 illustrates the stress-strain curves obtained from uniaxial deformation simulations conducted for different CNT weight fractions using the reactive force-field parameters reported by Singh et al. [26]. Although the strain rates implemented in the MD simulations are in the order of $10^{8}-10^{9} \mathrm{~s}^{-1}$, the incorporation of intermediate equilibration phases during the tensile deformation enables the relaxation of the system and long term averaging of stresses, thus making the stress-strain response more representative of a quasi-static experimental test. This is because, even as the bonds stretch from their equilibrium state, the system continues to be equilibrated simultaneously. This phenomenon cannot be verified and resolved experimentally; however, the molecular simulations provide insights into key trends defining plasticity while taking into account both the interaction between CNT and polymer matrix and influence of different weight fraction of CNT. The stress-strain curve indicates a high tensile modulus in the elastic region, followed by a significant plastic hardening region. As the weight fraction of the CNT in the polymer increases, the slope of the hardening phase in the stress-strain curve also increases. The observed plastic hardening could lead to an increase in fracture toughness. The tensile modulus for the polymer RUCs with different CNT weight fractions varies from $64 \mathrm{GPa}$ for $1 \% \mathrm{CNT}$ to $91 \mathrm{GPa}$ for $5 \% \mathrm{CNT}$.

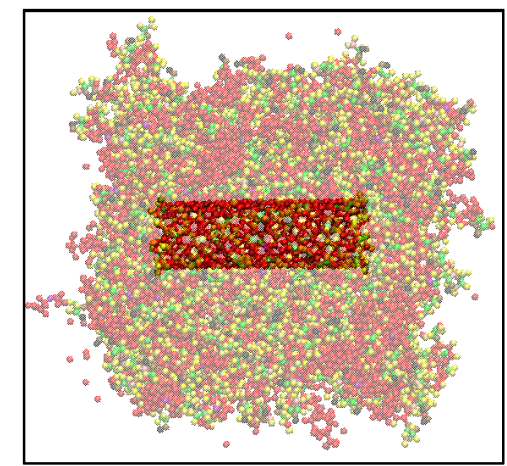

Figure 5. Ideal RUC with CNT oriented along the direction of load 


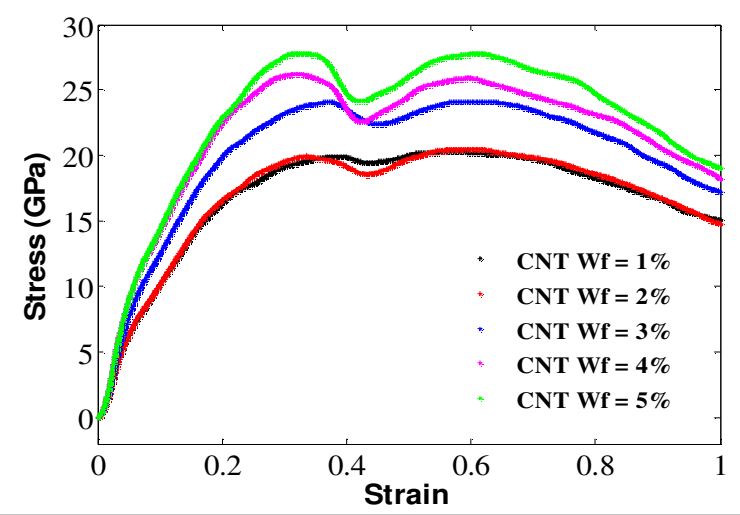

Figure 6. Stress-strain curves for CNT-epoxy system using ReaxFF

The addition of CNT molecules to the epoxy system not only alters its mechanical response but also affects the probability of cross-link formation between the resin and the hardener. It is important to note that the orientation of a single CNT affects the load carrying capacity of the RUC in that direction; hence, a system with randomly distributed and oriented CNTs is generated to investigate the effect of the nanoinclusions by weight. Using these stochastic models, a larger range of CNT weight fractions are studied to understand their effect on elastic mechanical properties. The variation of mechanical properties with changes in CNT weight fraction is shown in Figure 7. Multiple simulations with random initial configurations pertaining to each CNT weight fraction are performed, and the corresponding variability in data is also shown in the form of error bars.

It can be inferred from Figure 7(a) that there is no significant improvement in the value of tensile modulus of the system at CNT weight fractions beyond 6\%. An improvement in the bulk modulus is observed due to the inclusion of CNTs in the polymeric phase up to a weight fraction of $10 \%$; beyond this weight percentage, the mechanical properties appear to degrade due to ineffective load transfer between molecules. The inclusion of CNTs also affects the formation of bonds between the resin and the hardener. At lower weight fractions, CNTs act as a barrier in space and reduce the probability of cross-linking. However, the gradient of reduction in cross-linking degree reduces with increase in weight fraction; at a CNT weight percentage of $15 \%$, there is a rise in effective bond formation. This is because the CNTs form a cluster at the center of the RUC due to their high intermolecular forces, and leave the remaining volume in the RUC available for the polymer to form bonds. This phenomenon of CNT clustering, 
however, leads to ineffective load transfer between molecules and a corresponding deterioration in mechanical properties.

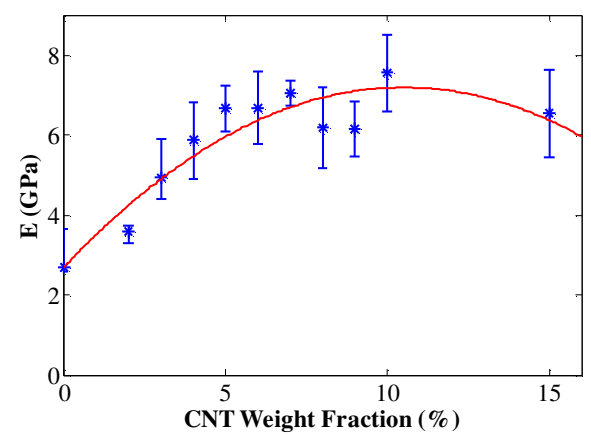

(a)

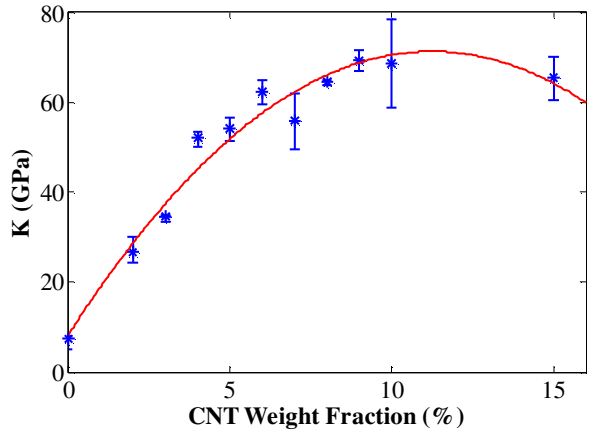

(b)

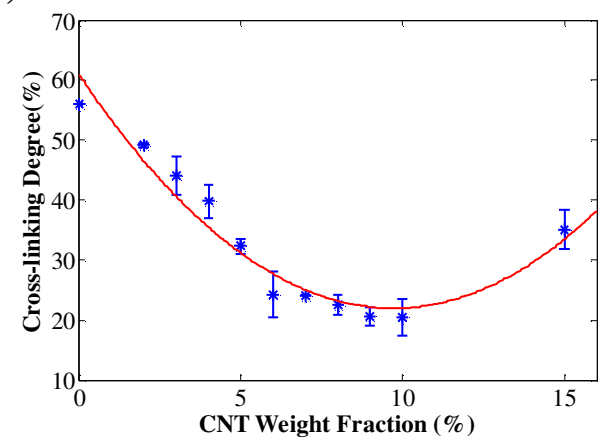

(c)

Figure 7. Variation of mechanical properties with CNT weight fraction (a) Tensile modulus (b) Bulk modulus (c) Cross-linking degree

The results clearly illustrate the deviation of mechanical properties from conventional homogenization techniques that assume a linear relationship between weight fraction and tensile modulus. The traditional Mori-Tanaka approximation was performed to evaluate the tensile modulus of the nanopolymer at various weight fractions, and the error between the MD results and the homogenized values was found to be $0.05 \%$ at $1 \%$ CNT weight fraction, $8.4 \%$ at $5 \% \mathrm{CNT}$ weight fraction, and $43.5 \%$ at $10 \% \mathrm{CNT}$ weight fraction in the nanopolymer. The high error margins reinforce the need for physics-based modeling approaches that capture fundamental physical and chemical interactions in the CNTenhanced epoxy polymer.

\section{Microscale Continuum Modeling}

A three dimensional (3D) stochastic microscale model is developed and implemented under periodic boundary conditions within a finite element (FE) framework using the commercial package ABAQUS 6.9. The micromechanics model accounts for the 
stochasticity in fiber placement and fiber geometry as well as the randomness in material properties of the polymer matrix dispersed with CNTs. Parameters pertinent to fiber geometry and matrix mechanical properties are sampled from relevant probability distributions in order to construct the micromechanical model. The uncertainty in the material properties of the fiber is assumed to follow a normal distribution based on standard tests and data presented in manufacturer datasheets [29]. The material properties of the polymer matrix are obtained from the nanoscale MD simulations performed in the current paper. The atomistic simulations provide a distribution of most likely material properties that serves as the input to the FE based micromechanics model. The effects of stochastic cross-linking degree and the corresponding changes in material properties due to the variation in cross linking degree are also incorporated into the microscale model. The local Young's modulus obtained from MD simulations and its relationship with the CNT weight fraction as shown in Figure 7(a) is established with a curve fit described in Equation (3), where $v_{C N T}\left(\mu_{C N T}, \sigma_{C N T}\right)$ is the probablistic function for the weight fraction of CNT given its mean $\left(\mu_{C N T}\right)$ and standard deviation $\left(\sigma_{C N T}\right)$, and $E_{C N T}$ is the Young's modulus of the CNT-dispersed epoxy section.

$$
E_{C N T}=-0.03837\left(v_{\mathrm{CNT}}\left(\mu_{C N T}, \sigma_{C N T}\right)^{2}\right)+0.95096\left(v_{\mathrm{CNT}}\left(\mu_{C N T}, \sigma_{C N T}\right)\right)+2.4459
$$

The PDF of the CNT weight fraction is assumed to be normally distributed and centered around a specific mean value with a standard deviation of 0.25 . Scanning electron microscope (SEM) images from reported literature on CNT-enhanced nanocomposites indicate that there can be large variations in the weight fraction of CNTs in the polymer due to agglomeration in certain areas; however, this effect is homogenized in the current work under the assumption that the CNTs in the nanopolymer are fairly well dispersed. Based on the results from MD simulations, it is clear that the crosslinking degree of the polymer matrix is also a function of the weight fraction of CNT inclusions in the polymer. The mathematical relationship between the amount of CNT inclusions and its effect on polymer network formation as seen in Figure 7(c) is formulated with a quadratic fit shown in Equation (4).

$$
\mu_{C D}=0.50056\left(v_{C N T}\left(\mu_{C N T}, \sigma_{C N T}\right)^{2}\right)-9.5905\left(v_{C N T}\left(\mu_{C N T}, \sigma_{C N T}\right)\right)+66.324
$$

In Equation (4), $\mu_{C D}$ corresponds to the mean cross-linking degree in the local region of the microscale model. The results from the MD simulation also confirm that the variation 
of cross-linking degree in a neat polymer (i.e., without CNT inclusions) is similar to a normal distribution. The variation of the Young's modulus with cross-linking degree for neat epoxy as seen in Figure 3(a) is described by a polynomial fit shown in Equation (5), where $C D$ is the cross-linking degree, and $E_{C D}$ represents the Young's modulus of the polymer corresponding to a specific cross-linking degree.

$$
E_{C D}=0.0159(C D)+1.566
$$

Furthermore, Equations (6) - (8) are employed to model the ratio of variations that characterizes a change in the Young's modulus due to variations in the cross-linking degree as well as the CNT weight fraction. $E_{\mu_{C D}}$ and $E_{C D_{p r o b}}$ correspond to the tensile modulus of the polymer at the mean cross-linking degree, and at a randomly sampled cross-linking degree, respectively. Each unit cell in the microscale continuum model contains a single fiber embedded in an epoxy matrix section. A representative volume element (RVE) for the model is generated by organizing multiple unit cells in a three dimensional array. By adopting a stochastic approach to the estimation of matrix properties, the epoxy section in each unit cell of the RVE is characterized by unique material properties, thus taking into account the local variations in the cross-linking degree and the CNT weight fraction using the probability distributions obtained from the MD simulations. Figure 8 illustrates the variations in the tensile modulus of 1000 epoxy matrix sections (i.e., RVE with $10 \times 10 \times 10$ unit cells) when the uncertainty in polymer cross-linking degree and CNT weight fraction is considered.

$$
\begin{gathered}
E_{\mu_{C D}}=0.0159\left(\mu_{C D}\right)+1.566 \\
E_{C D \text { prob }}=0.0159\left(C D\left(\mu_{C D}, \sigma_{C D}\right)\right)+1.566 \\
E_{\text {section }}=E_{C N T} \frac{E_{C D \text { prob }}}{E_{\mu_{C D}}}
\end{gathered}
$$




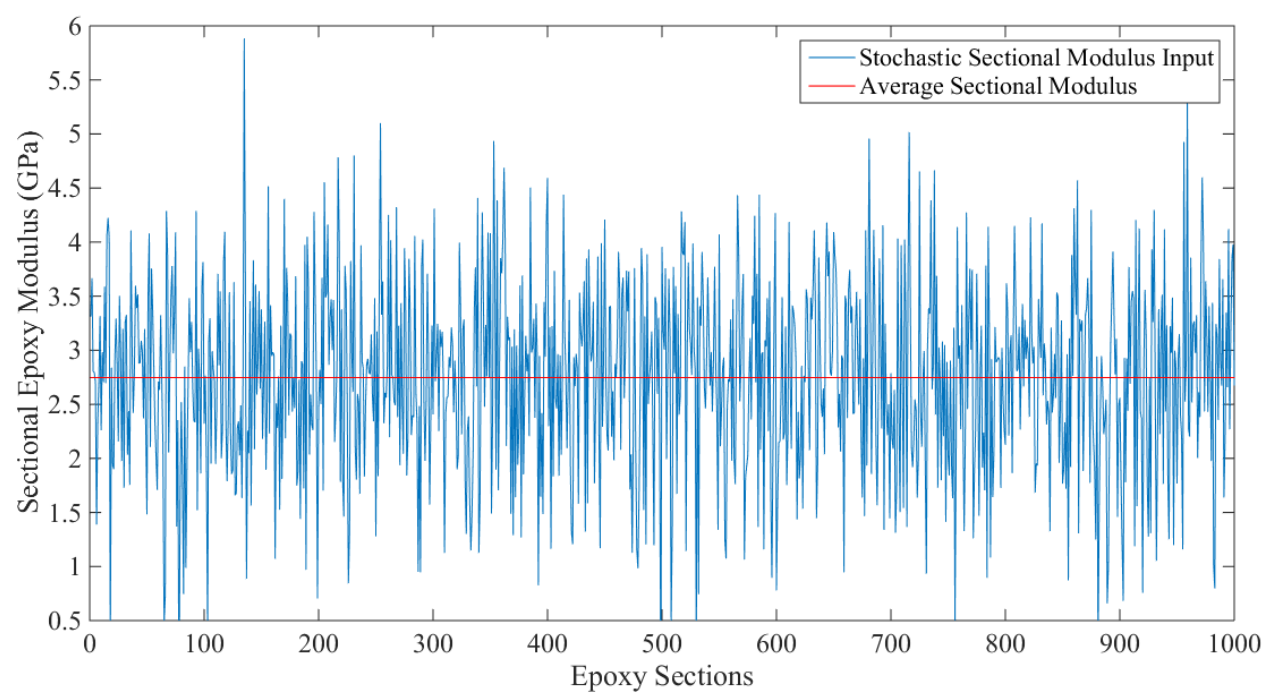

Figure 8. Variation in epoxy matrix material property with 1000 epoxy sections

The fiber is modeled as a perfectly elastic orthotropic material with mechanical properties extracted from IM7 fiber datasheets [29]. The micromechanical model determines the effective material property of the microscale volume element by applying a small displacement load and calculates the effective resistance of the volume element to the applied displacement. The model also assumes perfect adhesion between the fiber and the matrix at the interface. The triply periodic RVE is constructed of several unit cells with randomly sampled matrix properties and fiber geometry. This constraint mitigates size effects arising from the arrangement of multiple fiber-matrix unit cells and significantly reduces computational cost when larger RVEs are used. The applied strain increment, according to Pellegrino et al. [30] and Seguardo et al. [31], can be written as an averaged strain increment and a periodic strain increment. The periodic boundary conditions on a volume $\partial \mathrm{V}$ can be implemented using Equation (9), where $u_{i}$ are the displacements at the boundary of the cell which lead to changes in the behavior of the cell and $u_{i}^{p}$ are the periodic displacements which do not play a part in global deformations. Equation (9) can be rewritten in a discretized form suitable for implementation in the FE framework as described in Equation (10) where the elements $D_{i j}$ correspond to the global strains and $P_{1}$ and $P_{2}$ represent a set of symmetrically opposite points on the RVE.

$$
\begin{gathered}
u_{i}=D_{i j} y_{j}+u_{i}^{p} u_{i}^{p} \quad \text { periodic on } \partial \mathrm{V} \\
u_{i}\left(P_{2}\right)-u_{i}\left(P_{1}\right)=D_{i j}\left(y_{j}\left(P_{2}\right)-y_{j}\left(P_{1)}\right)\right.
\end{gathered}
$$


In order to apply the Equations (9) and (10) to the current micromechanical model, the geometry is meshed using a swept mesh control system. This meshing technique enables the nodes on opposite faces of the RVE to be ordered symmetrically thereby simplifying the application of the equations. The periodic boundary constraint equations are then applied to every nodal pair along all three degrees of freedom using fictitious reference points for each face. The mesh for the FE analysis consists of hexahedral elements, and its complexity depends on the fiber placement in each unit cell. For a seeding size of $0.6 \mu \mathrm{m}$ along the fiber direction, 83,600 elements are created, and for a seeding size of $0.95 \mu \mathrm{m}$ perpendicular to the fiber direction ( $E_{2}$ directed model), 28,584 elements are formed. The fully meshed microscale RVE imposed with periodic boundary conditions along all three dimensions is shown in Figure 9. The results from the convergence study demonstrating the tensile modulus convergence for different element edge lengths are illustrated in Figure 10.
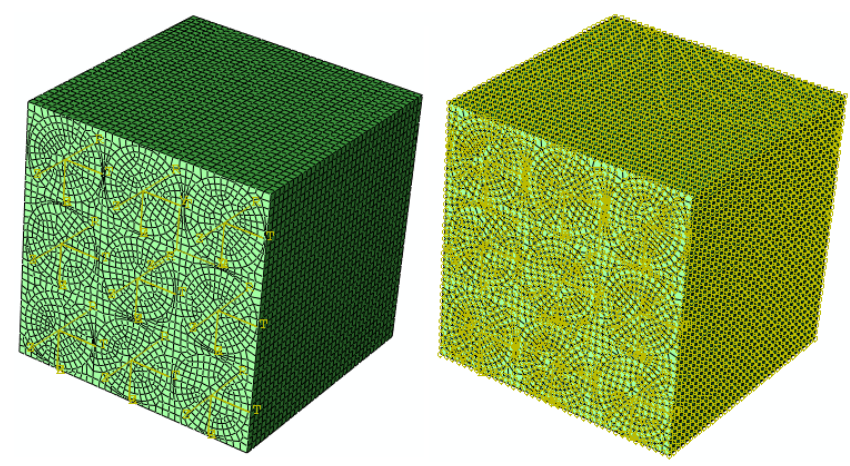

Figure 9. Fully meshed RVE with periodic boundary conditions applied as constraint equations

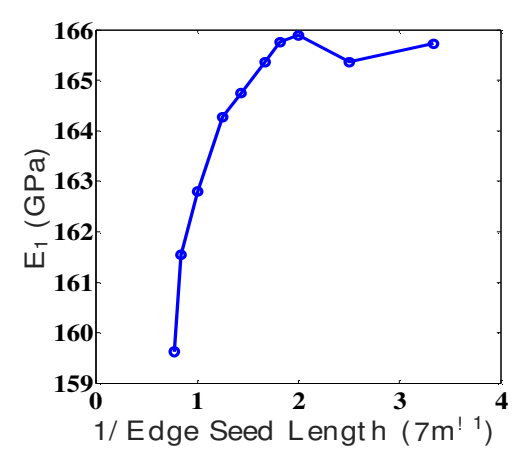

(a)

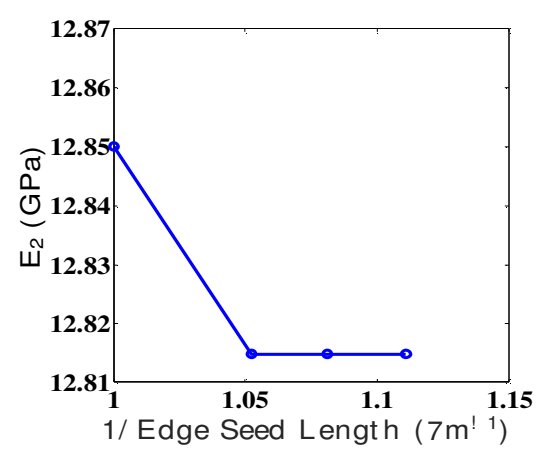

(b)

Figure 10. Tensile modulus convergence (a) along fiber direction (b) perpendicular to fiber direction 
Following an extensive mesh convergence study to determine the optimum number of elements required, the microscale continuum model is validated with material data from available literature for a system containing IM7 fibers and the 8552 epoxy system [32]. Figure 11 shows a comparison of stress contours from the validation study performed on an RVE with $4 \times 4 \times 4$ unit cells with perfectly ordered geometry and an RVE of similar size with microstructure variability. It is evident from the stress contours that accounting for microstructural variability leads to redistribution of stresses and regions with very low polymer cross-linking degree could serve as stress concentration hot spots. The validation study utilizes a deterministic fiber volume fraction value of 0.6 and no CNT inclusion in the matrix and the resulting effective modulus of the IM7/8552 composite system is compared to available literature. The deviation is estimated to be within acceptable tolerance for both $E_{1}$ and $E_{2}$, and the results from the validation study for the microscale continuum model are organized in Table 3.

Table 3. Comparison of tensile moduli of IM7/8552 composite system with literature data

\begin{tabular}{|c|c|c|c|}
\hline Tensile Modulus & $\begin{array}{c}\text { Value from } \\
\text { Literature }(\mathrm{GPa})\end{array}$ & $\begin{array}{c}\text { Model Prediction } \\
(\mathrm{GPa})\end{array}$ & $\begin{array}{c}\text { Error } \\
(\%)\end{array}$ \\
\hline $\begin{array}{c}\mathrm{E}_{1}(\text { Fiber } \\
\text { Direction) }\end{array}$ & $164\left(\sigma_{\mathrm{dev}}=23.7\right)$ & 165.3595 & 0.83 \\
\hline $\begin{array}{c}\mathrm{E}_{2} \text { (Transverse } \\
\text { Direction) }\end{array}$ & $12\left(\sigma_{\mathrm{dev}}=1.7\right)$ & 12.81481 & 6.79 \\
\hline
\end{tabular}
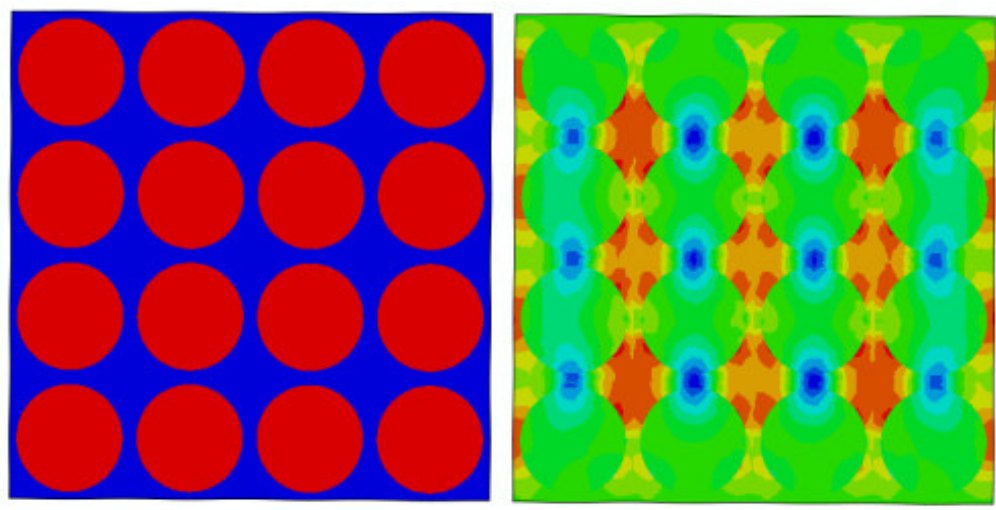

(a) 

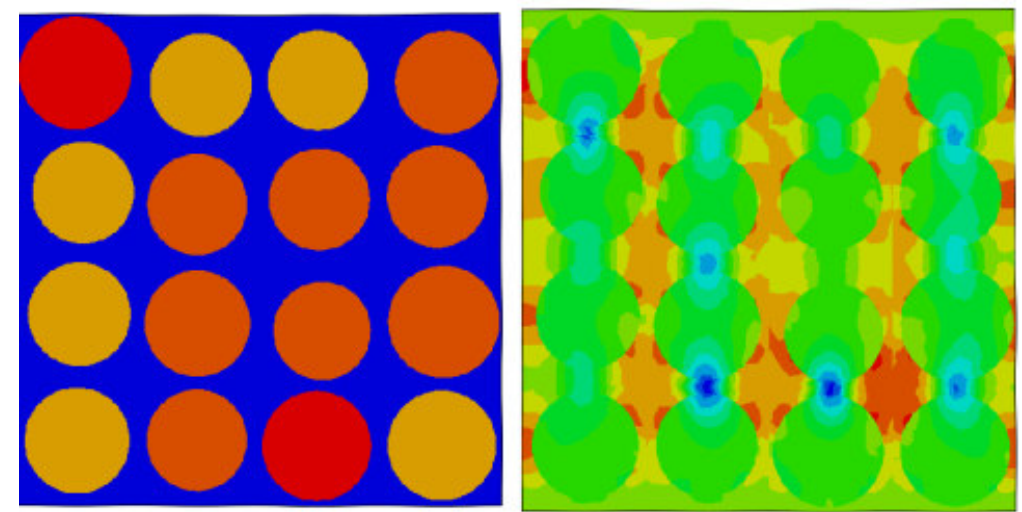

(b)

Figure 11. Stress maps from microscale model (a) perfectly ordered microstructure $(b)$ stochastic microstructure

Subsequent to the validation of the microscale continuum model for IM7/8552 system, stochasticity in fiber volume fraction and fiber geometry is introduced into the model; the uncertainty is modeled based on results from confocal microscopy performed on an AS4/EPON 832 laminate microstructure. The AS4/EPON 832 sample was manufactured using a hot press; hence, the consolidation of the fibers is high in the laminate. The distributions of the stochastic parameters are found to be near-Gaussian in nature, and a $\mathrm{KS}$ test is performed to confirm and justify the normality of the distributions. The characteristic parameters of the Gaussian distributions that define the uncertainty in fiber volume fraction and fiber diameter calculated from the microstructural study are tabulated in Table 4. It is important to note that the introduction of stochasticity in fiber geometry and volume fraction results in fewer degrees of randomness than the stochasticity in matrix properties; this is essentially because the fiber diameter and volume fraction are held constant in all sections along the length of a single fiber.

Table 4. Parameters of the Gaussian distributions for stochastic variables

\begin{tabular}{|c|c|c|}
\hline Property & Mean & $\sigma_{\mathrm{dev}}$ \\
\hline Fiber Volume Fraction & $71.75 \%$ & $4.67 \%$ \\
\hline Fiber Diameter & $6.99 \mu \mathrm{m}$ & $0.3209 \mu \mathrm{m}$ \\
\hline
\end{tabular}

The stochastic microscale continuum model is generated using an algorithm that perturbs the values of the stochastic variables from their mean values for every run. The sampling of variations using a Gaussian sampling scheme from the PDFs enables every run to contain unique values pertaining to matrix material property and fiber geometry. This algorithm is implemented to generate multiple microscale RVEs to effectively 
capture microstructural variability in the composite material system. The homogenized material properties obtained from the FE simulations are compiled to generate the variational histograms shown in Figure 12. Each histogram contains results from over 600 runs of the stochastic microscale continuum model with independently perturbed variables. Studies are performed on microscale RVEs with sections of neat epoxy surrounding fibers of variable diameters and volume fraction and RVEs containing fibers embedded in CNT-enhanced polymer. In the cases with CNT-enhanced polymer, the weight fraction of the CNT in each section of the matrix phase is sampled around a specific mean value $(1 \%$ or $3 \%)$.

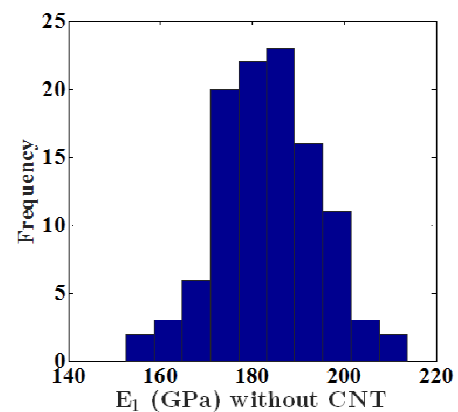

(a)

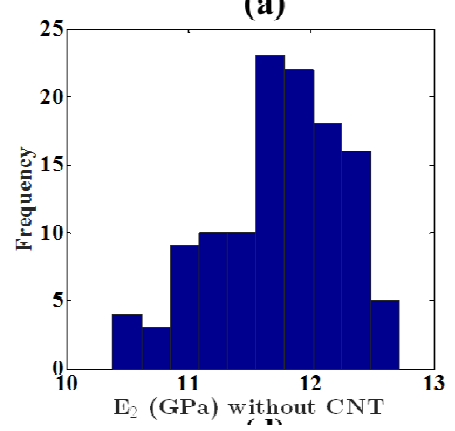

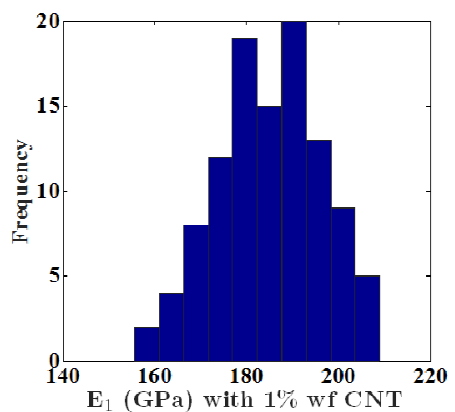

(D)

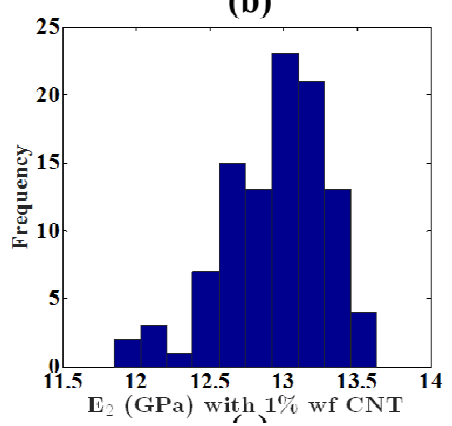

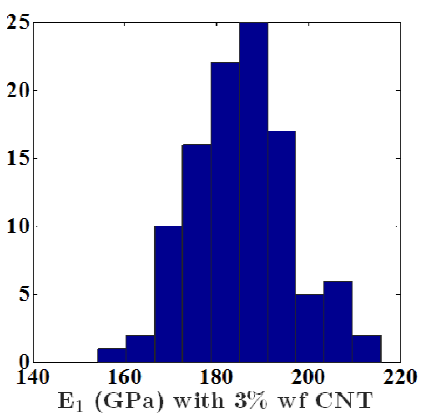

(c)

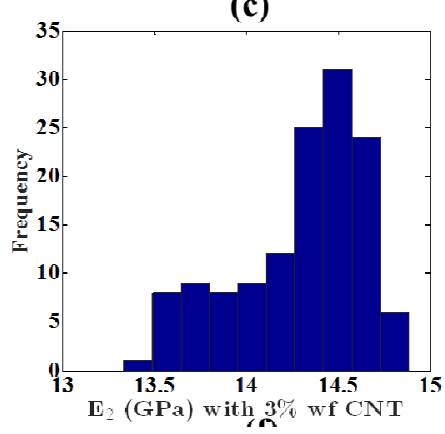

Figure 12. Histograms of homogenized material properties at the microscale (a) $E_{1}$ for RVE without CNT in matrix (b) $E_{1}$ for RVE with mean $1 \%$ CNT in matrix $(c) E_{1}$ for RVE with mean $3 \%$ CNT in matrix (d) $E_{2}$ for RVE without CNT in matrix (e) $E_{2}$ for $R V E$ with mean $1 \%$ CNT in matrix $(f) E_{2}$ for $R V E$ with mean $3 \%$ CNT in matrix

As expected, the inclusion of CNTs in the matrix phase does not cause a significant change in the expected value of the tensile modulus along the fiber direction as shown in Figures 12(a) to 12(c). The average improvement in the tensile modulus of the nanocomposite along the fiber direction is $0.77 \%$ for $1 \% \mathrm{CNT}$ weight fraction in the matrix, and $1.04 \%$ for $3 \% \mathrm{CNT}$ weight fraction. This validates the hypothesis that the fibers carry most of the load elastically; however, there is a $10.4 \%$ increase in the average transverse modulus of the RVE with an addition of $1 \% \mathrm{CNT}$ by weight, and a $22 \%$ 
increase in transverse modulus of the RVE with an addition of 3\% CNT by weight to the matrix phase. Along the transverse directions, the matrix tends to be the major load carrying constituent; hence, the addition of CNTs has a significant stiffening effect on the entire composite material system. It is interesting to note that the tensile modulus obtained from a few simulations containing a mean value $1 \% \mathrm{CNT}$ by weight in the matrix is lower than the maximum transverse tensile modulus obtained for the RVE containing neat epoxy. This overlap of values shows there is a distinct probability of achieving a lower transverse tensile modulus in some samples even with an increase in CNT weight fraction in the matrix. With the data obtained from the stochastic microscale simulations, a simple MC calculation compares the effect of CNT addition to the matrix on the entire composite material system; Equations (11) and (12) estimate the probability, $P$ (Lower $E_{2}$ ), of achieving a lower transverse tensile modulus despite the addition of higher weight percentage of CNT to the epoxy matrix. The parameters that characterize the histograms shown in Figure 12, obtained from the stochastic microscale continuum model, are organized in Table 5.

$$
\begin{gathered}
P_{1}\left(\text { Lower } E_{2}\right)=\frac{E_{2}(\text { Mean } 1 \% \text { Weight Fraction } C N T)}{\operatorname{Max}(\text { E2 }(\text { No } C N T))}=0.2451 \\
P_{2}\left(\text { Lower } E_{2}\right)=\frac{E_{2}(\text { Mean } 3 \% \text { Weight Fraction } C N T)}{\text { Max }(1 \% \text { Weight Fraction } C N T)}=0.0526
\end{gathered}
$$

Table 5. Results from stochastic microscale continuum model

\begin{tabular}{|c|c|c|c|c|}
\hline $\begin{array}{c}\text { Weight fraction } \\
\text { CNT in matrix }(\%)\end{array}$ & $\begin{array}{c}\text { Mean } \mathrm{E}_{1} \\
(\mathrm{GPa})\end{array}$ & $\begin{array}{c}\sigma_{\text {dev }} \text { in } \mathrm{E}_{1} \\
(\mathrm{GPa})\end{array}$ & $\begin{array}{c}\text { Mean } \mathrm{E}_{2} \\
(\mathrm{GPa})\end{array}$ & $\begin{array}{c}\sigma_{\text {dev }} \text { in } \mathrm{E}_{2} \\
(\mathrm{GPa})\end{array}$ \\
\hline 0 & 183.4 & 11.483 & 11.73 & 0.5179 \\
\hline 1 & 184.8 & 11.511 & 12.95 & 0.3556 \\
\hline 3 & 185.3 & 10.783 & 14.31 & 0.3499 \\
\hline
\end{tabular}

The second part of the microscale continuum study involves the characterization of plastic behavior in CNT-enhanced nanocomposites. MD simulations with bond order based reactive force field are used to obtain stress-strain data and characterize the strain hardening phase beyond the yield point of the nanopolymer as shown in Figure 5. This stress-strain curve is used to define the material properties of the micromechanical model where plasticity is defined explicitly based on the physics arising at the atomistic length scale. In this sense, the plastic behavior at the microscale is derived from the fundamental nonlinear extension and dissociation of bonds in the nanopolymer. MD simulations provided nonlinear stress strain curves for neat epoxy and CNT-enhanced nanopolymer 
containing $1 \%, 2 \%, 3 \%, 4 \%$, and $5 \%$ CNTs by weight fractions. However, the stochastic microscale model generates samples containing intermediate values of CNT weight fraction from the Gaussian distribution. Hence, the curves are interpolated to obtain stress-strain data for each of the specific sampled CNT weight fraction cases. The interpolation of the MD stress-strain curves is performed as demonstrated by Pellegrino et al. [30] using the assumption that all the interpolation points on different loading paths are characterized by the same number of steps, and they fall on the same plastic surface (i.e., at each $x$ value of the curve, the $y$ values are linearly interpolated for intermediate CNT weight fractions). Hence, a discrete stress response is obtained for every precise strain increment to the microscale RVE.

The nonlinear microscale continuum model is implemented to obtain a reliable comparison between a perfectly deterministic system (with ordered fibers and no variability in matrix properties) and a stochastic system (accounting for uncertainty in fiber geometry and matrix properties). Figure 13 illustrates the comparison of the plastic response for a perfectly ordered model and a stochastic model. Along the transverse direction, over $15 \mathrm{FE}$ simulations are performed for each model with a certain mean weight percentage of CNT in the matrix phase. However, along the fiber direction, the number of simulations was limited to five for each model due to the high computational cost incurred for convergence. 

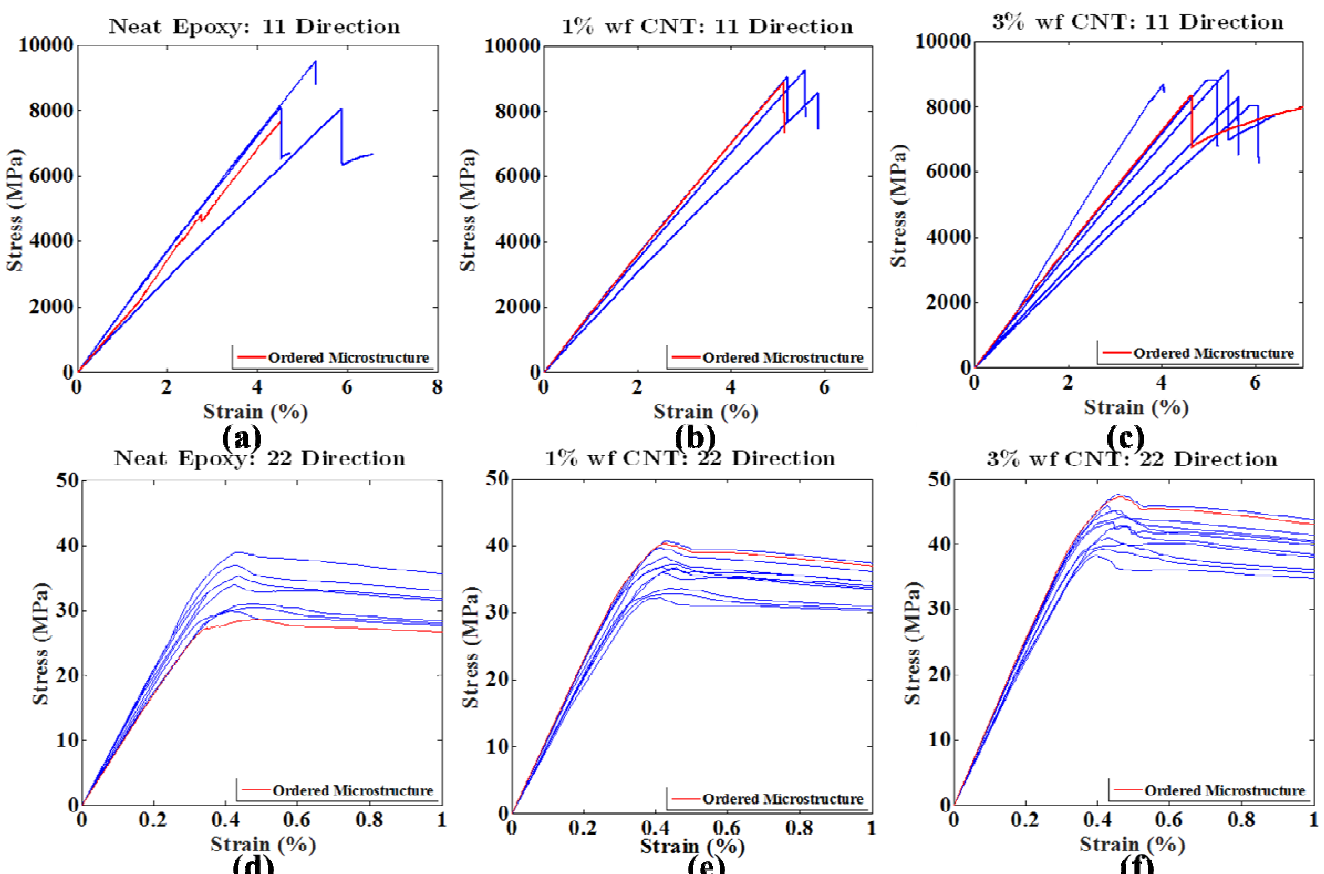

Figure 13. Stress-strain curves (a) along fiber direction with neat epoxy (b) along fiber direction with mean $1 \%$ CNT in matrix (c) along fiber direction with mean $3 \%$ CNT in matrix $(d)$ along transverse direction with neat epoxy (e) along transverse direction with mean $1 \%$ CNT in matrix (f) along transverse direction with mean 3\% CNT in matrix

Along the fiber direction, the majority of loads applied to the RVE are carried by the fiber and a sudden load drop is observed in Figure 13(a - c) as a result of matrix cracking (the fiber is modeled to be elastic) at the highest stress value. The load drops along the fiber direction are sudden; this confirms the hypothesis that the plasticity in the matrix does not cause any significant strain hardening along the fiber direction. The RVE with a perfectly ordered microstructure exhibited a higher limit in the stress-strain curve implying that a uniform microstructure would require the highest amount of energy to induce deformation. Any inclusion of variability in the RVE causes a reduction in the total energy absorption by the system thereby decreasing its resistance to deformation. The inclusion of CNTs does not significantly improve the load carry capacity of the nanocomposite beyond the yield point, and these results are well in agreement with the elastic simulations along the fiber direction which also showed that CNT inclusions resulted in a very moderate increase in the tensile modulus of the system.

The matrix is the active load carrying constituent in the transverse directions and the response is consistent with that of a ductile plastic system. The nonlinearities in the stress-strain curves occur at much lower stresses compared to the $\mathrm{E}_{1}$ simulations, and the 
drop in stresses is relatively gradual as seen in Figures $13(\mathrm{~d}-\mathrm{f})$. This result indicates that the activation of plasticity effects in the matrix occurs at a lower stress value. It can also be observed that the stress-strain curve flattens out at $\sim 0.4 \%$ strain which generally does not occur in experiments at the structural scale. The discrepancy between the microscale model results and experimental results at the structural scale could arise from the interaction of different scales resulting in further strain hardening at higher length scales, or from the assumption of perfect bonding at the fiber-matrix interface imposed in the model. The effects of a uniform microstructure is similar to the observations in the previous section involving linear elastic simulations; perfectly ordered systems show higher energy absorption compared to nonuniform RVEs. CNT dispersion in the matrix leads to an appreciable increase in energy absorption and as the weight percentage of CNTs in the matrix is increased, the critical strain after which the stress-strain curve flattens out, also increases. Another critical aspect to be noted is the considerable overlap in the stress-strain curve between each of these microscale RVEs where the response from a simulation with a lower weight percentage of CNT has a higher total strain energy value than the response from a simulation with lower weight percentage of CNT. These overlaps clearly support the need for a stochastic framework rather than a deterministic framework for the study of advanced nanocomposite systems.

\section{Conclusion}

Atomistic simulations were performed to study the behavior of CNT-dispersed epoxy polymer by characterizing the elastic-plastic response under tensile and triaxial loading conditions. A bond order based force-field was employed to investigate the deformation of bonds away from their equilibrium bond lengths caused by high strains. The maximum load carrying capacity of the CNTs in the matrix was estimated using an ideal RUC with the CNT aligned along the loading direction. Furthermore, stochastic RUCs containing CNTs of random distribution and orientation were analyzed and the corresponding mechanical properties were calculated. The fiber-centered microscale model utilized published values of material properties for the fiber obtained from manufacturer datasheets whereas the distributions for fiber diameter and volume fraction were characterized through experimental microstructural studies. The elastic-plastic response and the stochastic distributions from the MD simulations were used as input to the matrix 
phase of the model at the microscale by implementing a standard data fit process. Samples were collected of the input parameters from the stochastic distributions and the corresponding local material property of the matrix was calculated from a curve fit of MD simulation data. Results indicate that the microscale model can effectively capture the elastic and plastic mechanical response of the nanocomposite system using accurate data from molecular level simulations without resorting to a simple homogenization approach. The material and architectural variability at the microscale is efficiently simulated using the Gaussian sampling process, and the effect of this variability on the overall elastic and plastic response is well understood from the results of this study. The information obtained from the physical and chemical phenomena occurring at the molecular level is transferred across length scales and the stochastic microscale model will serve as an excellent bridging platform to understand the effects that the nanoparticles impart to the bulk properties of advanced nanocomposites at the structural scale.

\section{Acknowledgement}

This research is supported by the Office of Naval Research (ONR), Grant number: N00014-14-1-0068. The program manager is Mr. William Nickerson.

\section{References}

[1] Thostenson, E. T., Ren, Z., and Chou, T., 2001, "Advances in the Science and Technology of Carbon Nanotubes and their Composites: A Review," Composites Science and Technology, 61(13) pp. 1899-1912.

[2] Yamamoto, N., Hart, A. J., Garcia, E. J., 2009, "High-Yield Growth and Morphology Control of Aligned Carbon Nanotubes on Ceramic Fibers for Multifunctional Enhancement of Structural Composites," Carbon, 47(3) pp. 551-560.

[3] Dean, D., Obore, A. M., Richmond, S., 2006, "Multiscale Fiber-Reinforced Nanocomposites: Synthesis, Processing and Properties," Composites Science and Technology, 66(13) pp. 2135-2142.

[4] Show, Y., Nakashima, T., and Fukami, Y., 2013, "Anticorrosion Coating of Carbon Nanotube/Polytetrafluoroethylene Composite Film on the Stainless Steel Bipolar Plate for Proton Exchange Membrane Fuel Cells," Journal of Nanomaterials, 2013(2514103) pp. 2.

[5] Cheng, Q., Wang, B., Zhang, C., 2010, "Functionalized Carbon $\square$ Nanotube Sheet/Bismaleimide Nanocomposites: Mechanical and Electrical Performance Beyond Carbon $\square$ Fiber Composites," Small, 6(6) pp. 763-767. 
[6] Ghosh, S., Lee, K., and Moorthy, S., 1995, "Multiple Scale Analysis of Heterogeneous Elastic Structures using Homogenization Theory and Voronoi Cell Finite Element Method," International Journal of Solids and Structures, 32(1) pp. 27-62.

[7] Zohdi, T. I., Oden, J. T., and Rodin, G. J., 1996, "Hierarchical Modeling of Heterogeneous Bodies," Computer Methods in Applied Mechanics and Engineering, 138(1) pp. 273-298.

[8] Hasan, Z., Chattopadhyay, A., and Liu, Y., 2014, "Multiscale Approach to Analysis of Composite Joints Incorporating Nanocomposites," Journal of Aircraft, pp. 1-12.

[9] Zheng, Q., and Du, D., 2001, "An Explicit and Universally Applicable Estimate for the Effective Properties of Multiphase Composites which Accounts for Inclusion Distribution," Journal of the Mechanics and Physics of Solids, 49(11) pp. 2765-2788.

[10] Fish, J., and Wagiman, A., 1993, "Multiscale Finite Element Method for a Locally Nonperiodic Heterogeneous Medium," Computational Mechanics, 12(3) pp. 164-180.

[11] Arab, B., and Shokuhfar, A., 2013, "Molecular Dynamics Simulation of CrossLinked Epoxy Polymers: The Effect of Force Field on the Estimation of Properties," Журнал Нано-Та Електронної Фізики, (5,№ 1 (1)) pp. 01013-1-01013-5.

[12] Subramanian, N., Rai, A., Datta, S., "A Multiscale Model Coupling Molecular Dynamics Simulations and Micromechanics to Study the Behavior of CNT-Enhanced Nanocomposites," AIAA 2015 Science and Technology Exposition.

[13] Koo, B., Liu, Y., Zou, J., 2014, "Study of Glass Transition Temperature (Tg) of Novel Stress-Sensitive Composites using Molecular Dynamic Simulation," Modelling and Simulation in Materials Science and Engineering, 22(6) pp. 065018.

[14] Valavala, P., and Odegard, G., 2005, "Modeling Techniques for Determination of Mechanical Properties of Polymer Nanocomposites," Rev.Adv.Mater.Sci, 9pp. 34-44.

[15] Li, C., and Strachan, A., 2011, "Molecular Dynamics Predictions of Thermal and Mechanical Properties of Thermoset Polymer EPON862/DETDA," Polymer, 52(13) pp. 2920-2928.

[16] Rahmat, M., and Hubert, P., 2011, "Carbon Nanotube-polymer Interactions in Nanocomposites: A Review," Composites Science and Technology, 72(1) pp. 72-84.

[17] Subramaniyan, A. K., and Sun, C., 2008, "Continuum Interpretation of Virial Stress in Molecular Simulations," International Journal of Solids and Structures, 45(14) pp. 4340-4346.

[18] Borkowski, L., Liu, K., and Chattopadhyay, A., 2013, "From Ordered to Disordered: The Effect of Microstructure on Composite Mechanical Performance," CMC: Computers, Materials \& Continua, 37(3) pp. 161-193.

[19] Huang, Y., Jin, K. K., and Ha, S. K., 2008, "Effects of Fiber Arrangement on Mechanical Behavior of Unidirectional Composites," Journal of Composite Materials, .

[20] Buryachenko, V., Pagano, N., Kim, R., 2003, "Quantitative Description and Numerical Simulation of Random Microstructures of Composites and their Effective Elastic Moduli," International Journal of Solids and Structures, 40(1) pp. 47-72.

[21] Plimpton, S., 1995, "Fast Parallel Algorithms for Short-Range Molecular Dynamics," Journal of Computational Physics, 117(1) pp. 1-19.

[22] Jorgensen, W. L., Maxwell, D. S., and Tirado-Rives, J., 1996, "Development and Testing of the OPLS all-Atom Force Field on Conformational Energetics and Properties of Organic Liquids," Journal of the American Chemical Society, 118(45) pp. 1122511236. 
[23] Van Duin, A. C., Dasgupta, S., Lorant, F., 2001, "ReaxFF: A Reactive Force Field for Hydrocarbons," The Journal of Physical Chemistry A, 105(41) pp. 9396-9409.

[24] Chenoweth, K., van Duin, A. C., and Goddard, W. A., 2008, "ReaxFF Reactive Force Field for Molecular Dynamics Simulations of Hydrocarbon Oxidation," The Journal of Physical Chemistry A, 112(5) pp. 1040-1053.

[25] Weismiller, M. R., Duin, A. C. v., Lee, J., 2010, "ReaxFF Reactive Force Field Development and Applications for Molecular Dynamics Simulations of Ammonia Borane Dehydrogenation and Combustion," The Journal of Physical Chemistry A, 114(17) pp. 5485-5492.

[26] Singh, S. K., Srinivasan, S. G., Neek-Amal, M., 2013, "Thermal Properties of Fluorinated Graphene," arXiv Preprint arXiv:1303.2258, .

[27] Mattsson, T. R., Lane, J. M. D., Cochrane, K. R., 2010, "First-Principles and Classical Molecular Dynamics Simulation of Shocked Polymers," Physical Review B, 81(5) pp. 054103.

[28] Wei, C., Cho, K., and Srivastava, D., 2001, "Chemical bonding of polymer on carbon nanotube," MRS Proceedings, Anonymous Cambridge Univ Press, 675, pp. W4. 7.1 .

[29] Hexcel, 2011, "HexTow IM7 Carbon Fibre Product Literature," Stamford, CT.

[30] Pellegrino, C., Galvanetto, U., and Schrefler, B., 1999, "Numerical Homogenization of Periodic Composite Materials with Non-Linear Material Components," International Journal for Numerical Methods in Engineering, 46(10) pp. 1609-1637.

[31] Segurado, J., and Llorca, J., 2002, "A Numerical Approximation to the Elastic Properties of Sphere-Reinforced Composites," Journal of the Mechanics and Physics of Solids, 50(10) pp. 2107-2121.

[32] Hexcel, 2011, "Hexcel HexPly 8552 Product Literature," Stamford, CT. 